University of Nebraska - Lincoln

DigitalCommons@University of Nebraska - Lincoln

Ralph Skomski Publications

Research Papers in Physics and Astronomy

$7-2013$

\title{
Magnetism of MnBi-Based Nanomaterials
}

\author{
Parashu Kharel \\ University of Nebraska-Lincoln, pkharel2@unl.edu \\ Shah R. Valloppilly \\ University of Nebraska-Lincoln, svalloppilly2@unl.edu \\ Ralph Skomski \\ University of Nebraska-Lincoln, rskomski2@unl.edu \\ Jeffrey E. Shield \\ University of Nebraska-Lincoln, jshield@unl.edu \\ David J. Sellmyer \\ University of Nebraska-Lincoln, dsellmyer@unl.edu
}

Follow this and additional works at: https://digitalcommons.unl.edu/physicsskomski

Kharel, Parashu; Valloppilly, Shah R.; Skomski, Ralph; Shield, Jeffrey E.; and Sellmyer, David J., "Magnetism of MnBi-Based Nanomaterials" (2013). Ralph Skomski Publications. 79.

https://digitalcommons.unl.edu/physicsskomski/79

This Article is brought to you for free and open access by the Research Papers in Physics and Astronomy at DigitalCommons@University of Nebraska - Lincoln. It has been accepted for inclusion in Ralph Skomski Publications by an authorized administrator of DigitalCommons@University of Nebraska - Lincoln. 


\title{
Magnetism of MnBi-Based Nanomaterials
}

\author{
P. Kharel ${ }^{1,2}$, V. R. Shah ${ }^{2}$, R. Skomski ${ }^{1,2}$, J. E. Shield ${ }^{2,3}$, and D. J. Sellmyer ${ }^{1,2}$ \\ ${ }^{1}$ Department of Physics and Astronomy, University of Nebraska, Lincoln, NE 68588 USA \\ ${ }^{2}$ Nebraska Center for Materials and Nanoscience, University of Nebraska, Lincoln, NE 68588 USA \\ ${ }^{3}$ Mechanical and Materials Engineering, University of Nebraska, Lincoln, NE 68588 USA
}

\begin{abstract}
Nanostructured MnBi ribbons doped with impurity elements including B, C, Fe, Hf, Sm and Tb were prepared using the arc melting and melt-spinning techniques. The melt-spun ribbons were annealed in vacuum furnace at $350^{\circ} \mathrm{C}$ to obtain the intended hexagonal structure. The external impurity doping made a significant change in the magnetic properties of the nanostructured MnBi ribbons including a decrease in saturation magnetization $\left(M_{s}\right)$ and anisotropy energy $(K)$ and an increase in coercivity $\left(H_{c}\right)$. However, $H f$ and $C$ co-doping showed the opposite effect with a small increase in both $M_{s}$ and $K$. Interestingly, the anisotropy energy of the boron doped sample increased by about $15 \%$ irrespective of the small decrease in magnetization. A significant increase in $H_{c}$ of $M_{n B i}$ ribbons was found due to $\mathrm{Hf}$, Tb and Sm doping. $\mathrm{H}_{\mathrm{c}}$ as high as $13 \mathrm{kOe}$ was achieved in Hf-doped sample after the sample was aligned in a magnetic field. A thermal hysteresis was observed at the structural phase transition of $\mathrm{MnBi}$, which shifts by about $5 \mathrm{~K}$ towards higher temperatures due to impurity doping. The observed magnetic properties of the impurity doped $\mathrm{MnBi}$ ribbons are explained as the consequences of the disorder and the competing ferromagnetic and antiferromagnetic interactions.
\end{abstract}

Index Terms - Magnetic anisotropy, melt-spun ribbons, permanent magnet.

\section{INTRODUCTION}

$\mathbf{T}$ HE non-rare-earth materials having high magnetic anisotropy and high Curie temperature are gaining significant attention because of increasing cost, irregular supply and increasing demand of some of the rare-earth elements which are essential for producing powerful permanent magnets [1], [2]. A manganese-based ferromagnetic material $\mathrm{MnBi}$ is one of such materials that has a high magnetic anisotropy $(\mathrm{K})$ of $16 \mathrm{Mergs} / \mathrm{cm}^{3}$ at room temperature and also has a high Curie temperature, well above room temperature. An interesting feature in the magnetic anisotropy of $\mathrm{MnBi}$ is that the value of $\mathrm{K}$ is negative below $100 \mathrm{~K}$ but increases with increasing temperature and attains its maximum value of $22 \mathrm{Mergs} / \mathrm{cm}^{3}$ at $490 \mathrm{~K}$ [3]. The high-temperature value of $\mathrm{K}$ is much higher than that of $\mathrm{Nd}_{2} \mathrm{Fe}_{14} \mathrm{~B}$ magnet, the most powerful (highest energy product) permanent magnet developed so far. This has made $\mathrm{MnBi}$ a material of interest for high-temperature permanent magnet applications. Other interesting properties of $\mathrm{MnBi}$ in thin films are the high value of room temperature perpendicular anisotropy [4], the high value of transport spin polarization [5] and an unusual spin correlations leading to a Kondo effect when doped with heavy and noble metals such as $\mathrm{Pt}$ and $\mathrm{Au}$ [6], [7].

Although magnetic anisotropy of $\mathrm{MnBi}$ is promising for permanent-magnet applications, the maximum energy product (7.1 MGOe at room temperature) that determines the strength of a magnet is relatively low for most practical applications [8]. This is because the theoretical limit of saturation magnetization for $\mathrm{MnBi}$ is about $712 \mathrm{emu} / \mathrm{cm}^{3}$, which is much smaller than that of the most other permanent magnet materials [6], [9]. Some of the other issues concerning the structural and magnetic properties of this material are (i) the structural phase transition before the Curie temperature is reached and (ii) the diffusion of $\mathrm{Mn}$ atoms into the empty interstitial sites causing the net magnetization

Manuscript received October 24, 2012; revised January 30, 2013; accepted February 01, 2013. Date of current version July 15, 2013. Corresponding author: P. R. Kharel (e-mail: pkharel2@unl.edu).

Color versions of one or more of the figures in this paper are available online at http://ieeexplore.ieee.org.

Digital Object Identifier 10.1109/TMAG.2013.2245497 to decrease. Also, $\mathrm{MnBi}$ is highly susceptible to moisture and oxygen contamination. Therefore, there have been extensive research efforts to increase the resistance to degradation, improve magnetic properties and form a phase that is stable and exhibits a high Curie temperature. These improvements are expected to be achieved by alloying $\mathrm{MnBi}$ with a suitable third element. Our interest is in the magnetic properties, where we have investigated the effect of external impurity doping on the saturation magnetization, coercivity, anisotropy energy and Curie temperature of the nanostructured MnBi ribbons. We have chosen six doping specimens; (i) a ferromagnetic transition metal (Fe) which has a high saturation magnetization (ii) two rare-earth elements; one $(\mathrm{Sm})$ having positive and the other $(\mathrm{Tb})$ having negative atomic quadrupole moments (iii) a $5 \mathrm{~d}$ element (Hf) where the induced magnetic moment is expected to be small and to couple with the manganese moments and (iv) two non-magnetic elements (B and C) which are expected to help in developing structural stability.

\section{EXPERIMENTAL MethodS}

Nanostrucutred MnBi:X (X = B, C, Fe, Hf, Sm and Tb) ribbons were prepared by arc-melting, melt-spinning and annealing. The arc-melting process, which produces MnBi:X ingots from the respective metal pieces, was carried out on a water cooled $\mathrm{Cu}$ hearth in a highly pure argon environment. In order to produce nanostructured ribbons, a molten mixture formed by melting MnBi:X ingot was ejected onto the surface of a rotating copper wheel where it rapidly solidified into ribbons. The tangential speed of the rotating wheel was kept at $40 \mathrm{~m} / \mathrm{s}$ for all the samples and the melt-spinning process was carried out in the melt-spinner chamber filled with a suitable pressure of highly pure argon gas. In order to obtain the intended crystal structure, the ribbons were annealed in a tubular vacuum furnace (base pressure $\sim 10^{-6}$ Torr) at $350^{\circ} \mathrm{C}$ for 4 hours. We also prepared powder samples by mechanically grinding the annealed ribbons in acetone. The elemental compositions of the samples as determined by energy dispersive $\mathrm{X}$-ray (EDX) spectroscopy are $\mathrm{Mn}_{54} \mathrm{Bi}_{46}, \mathrm{Mn}_{53} \mathrm{Fe}_{4} \mathrm{Bi}_{43}$, $\mathrm{Mn}_{47} \mathrm{Hf}_{3} \mathrm{Bi}_{50}, \mathrm{Mn}_{51} \mathrm{Sm}_{4} \mathrm{Bi}_{45}$ and $\mathrm{Mn}_{54} \mathrm{~Tb}_{4} \mathrm{Bi}_{42}$. Because of the big difference in the melting points of $\mathrm{Mn}$ and $\mathrm{Bi}$, it is very difficult to control the elemental composition during melt spinning. Since the content of B and C could not be 


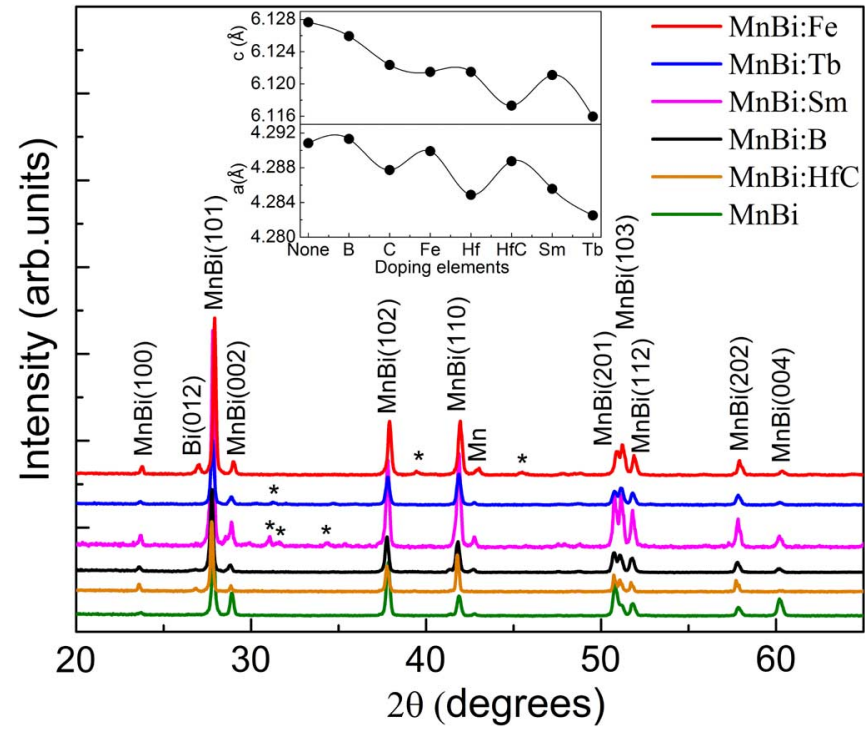

Fig. 1. Room temperature $\mathrm{X}$-ray diffraction patterns of $\mathrm{MnBi}: \mathrm{X}(\mathrm{X}=\mathrm{Fe}, \mathrm{Tb}$, $\mathrm{Sm}, \mathrm{B}, \mathrm{HfC}$ ) ribbons. The base lines are shifted for clarity and the patterns are arranged in the order of the legend shown at the top right corner of the figure, where the pattern for $\mathrm{MnBi}$ is at the bottom and that for MnBi:Fe is at the top. The star symbols correspond to Bragg reflections from elemental $\mathrm{Bi}$. The inset shows the lattice parameters as a function of impurity doping.

determined by EDX spectroscopy, we estimated the amount of these elements from their weights in the starting mixture, which is about $1 \%$. One of the samples we studied was co-doped with $\mathrm{Hf}$ and $\mathrm{C}(2 \% \mathrm{Hf}$ and $1 \% \mathrm{C})$. The structural properties of the samples were studied using X-ray powder diffraction and the magnetic properties were investigated using a Quantum Design Magnetic Properties Measurement System (MPMS) and Physical Properties Measurement System (PPMS).

\section{RESUlTS AND DISCUSSION}

As shown in Fig. 1, the most intense peaks in the roomtemperature $\mathrm{x}$-ray diffraction (XRD) patterns of the $\mathrm{MnBi}$ and $\mathrm{MnBi}: \mathrm{X}(\mathrm{X}=\mathrm{Fe}, \mathrm{Tb}, \mathrm{Sm}, \mathrm{B}, \mathrm{HfC})$ ribbons are indexed based on the hexagonal NiAs crystal structure. The diffraction patterns indicate that the impurity doping has not produced a significant change in the lattice symmetry but there is a noticeable change in both the $c$ and $a$ lattice parameters (see the inset of Fig. 1). The lattice parameters were calculated from the Rietveld analysis of the powder x-ray diffraction data. However, refinement of fractional occupancy of the different atomic species was excluded in this analysis. As a consequence of preferential orientation of crystallites and possible fractional occupation of dopant atoms at the crystallographic sites, there is a slight deviation of the experimental x-ray diffraction pattern with respect to the calculated diffraction pattern. However, the accuracy of lattice parameters is not influenced by this mismatch and the variation of cell parameters is consistent with the assumption that a fraction of the dopant atoms may have been incorporated into the $\mathrm{MnBi}$ lattice. Although there are reflections from elemental impurities, no indications of secondary alloy phases have been found within the limit of $\mathrm{x}$-ray diffraction. All the $\mathrm{MnBi}$ :X ribbons are composed of nanocrystalline grains but the grain sizes are different for the ribbons of different elemental compositions. The grain sizes as determined using the Scherrer's formula are between $27 \mathrm{~nm}$ and $43 \mathrm{~nm}(27 \mathrm{~nm}$ for MnBi:HfC and $43 \mathrm{~nm}$ for

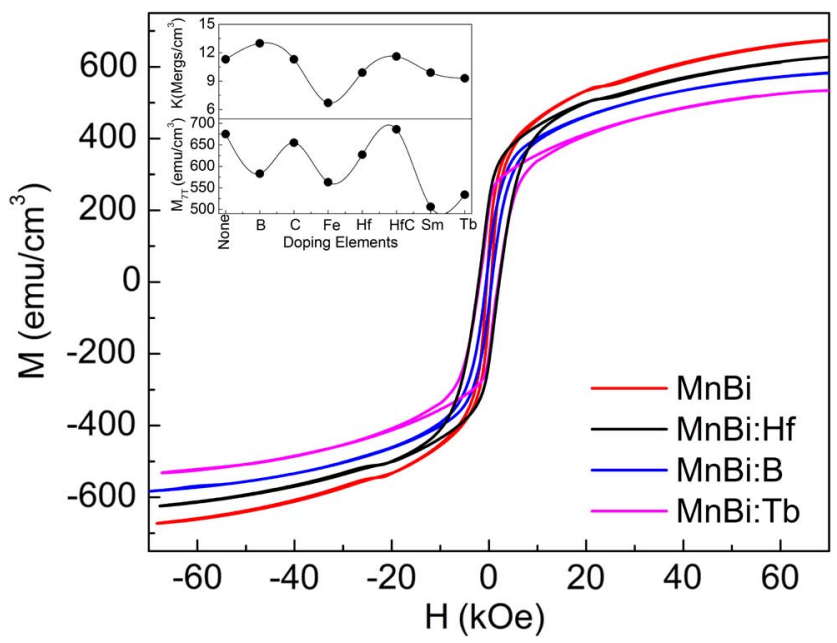

Fig. 2. Magnetic field dependence of magnetization $\mathrm{M}(\mathrm{H})$ for $\mathrm{MnBi}, \mathrm{MnBi}: \mathrm{Hf}$, $\mathrm{MnBi}: \mathrm{B}$ and $\mathrm{MnBi}: \mathrm{Tb}$ ribbons. The upper and lower panels in the inset respectively show how magnetic anisotropy $(\mathrm{K})$ and saturation magnetization $\left(\mathrm{M}_{7 \mathrm{~T}}\right)$ change as $\mathrm{MnBi}$ is doped with different elements. Lines connecting the data points in the inset are just guides to the eye.

$\mathrm{MnBi}: \mathrm{Sm}$ ) showing that the grain size is sensitive to the nature of the dopant elements. We note that the ribbons were prepared under similar synthesis and annealing conditions.

The magnetic properties of the MnBi ribbons are highly sensitive to the external impurity doping. Although some of the samples contain a small amount of elemental $\mathrm{Mn}$ and $\mathrm{Bi}$, the contribution from these elements to the observed magnetic properties can be considered to be negligibly small. Fig. 2 shows the $\mathrm{M}(\mathrm{H})$ hysteresis loops for four representative samples, namely, MnBi, MnBi:Hf, MnBi:B and MnBi:Tb. All the samples show strong ferromagnetic moment at room temperature but the $\mathrm{M}(\mathrm{H})$ loops are not saturated even at 7 T. At $7 \mathrm{~T}$, we have measured a large room-temperature magnetization of about $675 \mathrm{emu} / \mathrm{cm}^{3}(75 \mathrm{emu} / \mathrm{g})$ in the undoped $\mathrm{MnBi}$ ribbons which is very close to the theoretical value of $712 \mathrm{emu} / \mathrm{cm}^{3}(80 \mathrm{emu} / \mathrm{g})$. Magnetization $(\mathrm{M})$, a parameter related to the strength of a magnet, showed significant decrease with impurity doping, irrespective of whether the dopants carried magnetic moment or not except in the case of $\mathrm{Hf}$ and $\mathrm{C}$ co-doping where magnetization showed moderate increase. As shown in the lower panel of the inset of Fig. 2, the high-field room-temperature magnetizations $\left(\mathrm{M}_{7 \mathrm{~T}}\right)$ recorded for $\mathrm{B}, \mathrm{C}$, $\mathrm{Fe}, \mathrm{Hf}, \mathrm{Sm}$ and $\mathrm{Tb}$ doped ribbons are 583, 655, 563, 627, 506 and $534 \mathrm{emu} / \mathrm{cm}^{3}$ respectively. However, the observed value of $\mathrm{M}_{7 \mathrm{~T}}\left(686 \mathrm{emu} / \mathrm{cm}^{3}\right)$ in $\mathrm{Hf}$ and $\mathrm{C}$ co-doped sample is little higher than the value measured in $\mathrm{MnBi}$ ribbon. In contrast to the magnetization, the anisotropy energy $(\mathrm{K})$ shows little increase above the level of $\mathrm{MnBi}\left(11 \mathrm{Mergs} / \mathrm{cm}^{3}\right.$ for $\mathrm{MnBi}$ and $13 \mathrm{Mergs} / \mathrm{cm}^{3}$ for MnBi:B) due to B doping. However, as shown in the upper panel of the inset of Fig. 2, there is a significant decrease (about $40 \%$ ) in the value of $\mathrm{K}$ due to $\mathrm{Fe}$ doping. $\mathrm{Tb}$ and $\mathrm{Sm}$ doping produced between 10 to $15 \%$ decrease in $\mathrm{K}$. The change in the value of $\mathrm{K}$ due to $\mathrm{Hf}$ and $\mathrm{C}$ is very small. The anisotropy energy was calculated using the approach to saturation method, where the high-field data was fitted to $\mathrm{M}=\mathrm{M}_{0}\left(1-\mathrm{A} / \mathrm{H}^{2}\right)+\chi \mathrm{H} ; \mathrm{A}=4 \mathrm{~K}^{2} / 15 M_{0}^{2}$. The parameters $\mathrm{M}_{0}, \mathrm{~A}$ and $\chi$ are the spontaneous magnetization, a constant that depends on $\mathrm{K}$ and the high field susceptibility, respectively [10]. 


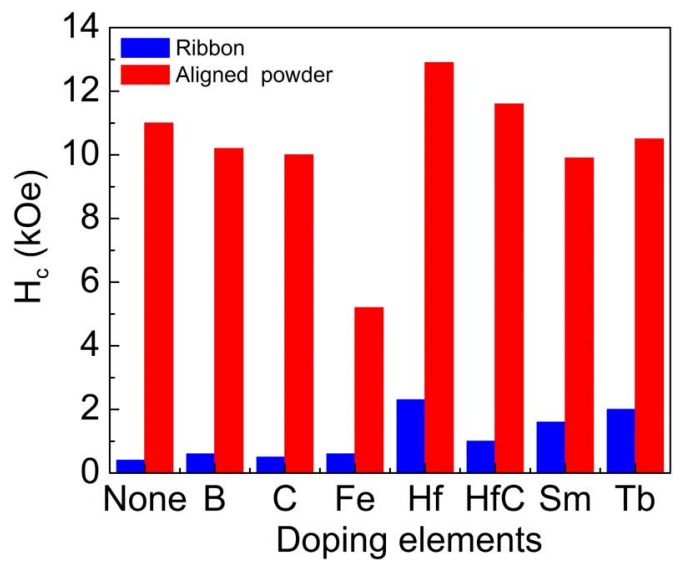

Fig. 3. Coercivities of $\mathrm{MnBi}$ and $\mathrm{MnBi} \mathrm{X}(\mathrm{X}=\mathrm{B}, \mathrm{C}, \mathrm{Fe}, \mathrm{Hf}, \mathrm{Sm}$ and $\mathrm{Tb})$ samples. The blue bars correspond to the coercivities of $\mathrm{MnBi}$ : $\mathrm{X}$ ribbons and the red bars show the coercivities after the crushed ribbons were aligned in a magnetic field.

As mentioned above, one of the issues related to $\mathrm{MnBi}$ is the interstitial occupation of Mn or dopant atoms. Interstitial Mn moments couple antiferromagnetically with the other Mn atoms causing the net magnetization to decrease [6]. The exact positions of the impurity atoms in the $\mathrm{MnBi}$ lattice are uncertain but the decrease in magnetization is consistent with the competing ferromagnetic and antiferromanetic interactions in the MnBi:X system. The small increase of magnetization in the $\mathrm{Hf}$ and $\mathrm{C}$ co-doped sample may indicate that the diffusion of $\mathrm{Mn}$ into the interstitial sites is relatively small in this case and also possibly there is a ferromagnetic coupling between Mn and Hf moments. Magnetocrystalline anisotropy of a compound depends on its electronic structure and hence on the crystal structure. Hence, the change in atomic arrangement due to external impurity doping may also change the value of anisotropy energy [11]. Since the signs of the atomic quadrupole moments for Sm and $\mathrm{Tb}$ are opposite, their contributions to the anisotropy energy are also expected to be opposite, provided that the doping does not make any change in the crystal lattice. In our case, such an opposite trend was not found suggesting a different source for the change in the anisotropy energy of the impurity-doped $\mathrm{MnBi}$ ribbons. It is likely that the large decrease in $\mathrm{K}$ in Fe doped sample is due to the interstitial disorder where Fe occupies the $\mathrm{Mn}$ sites, pushing more $\mathrm{Mn}$ into the interstitial sites [12]. However, boron, that has a lot smaller atomic radius compared to that of $\mathrm{Mn}$ and $\mathrm{Bi}$, may occupy vacant interstitial sites reducing the diffusion of Mn onto those sites leading to anisotropy energy to increase.

As shown in Fig. 3, there is a substantial increase in the value of $\mathrm{H}_{\mathrm{c}}$ as the $\mathrm{MnBi}$ ribbons are doped with heavy elements including $\mathrm{Hf}, \mathrm{Sm}$ and $\mathrm{Tb}$ but the change is relatively small for $\mathrm{B}, \mathrm{C}$ and Fe doping. Basically, coercivity of a magnet depends on the magnetic anisotropy and also on the microstructure of the sample. In our case, there is an apparent change in both the grain size and the anisotropy energy of the $\mathrm{MnBi}$ ribbons due to impurity doping but this change does not directly correlate with the observed change in coercivity. However, since the samples doped with heavy elements show larger coercivities, the role of magnetic anisotropy cannot be completely ruled out. Furthermore, MnBi shows a high perpendicular anisotropy in thin films and the isotropic ribbons prepared at high wheel speed $(40 \mathrm{~m} / \mathrm{s})$ are not expected to show very high coercivity.

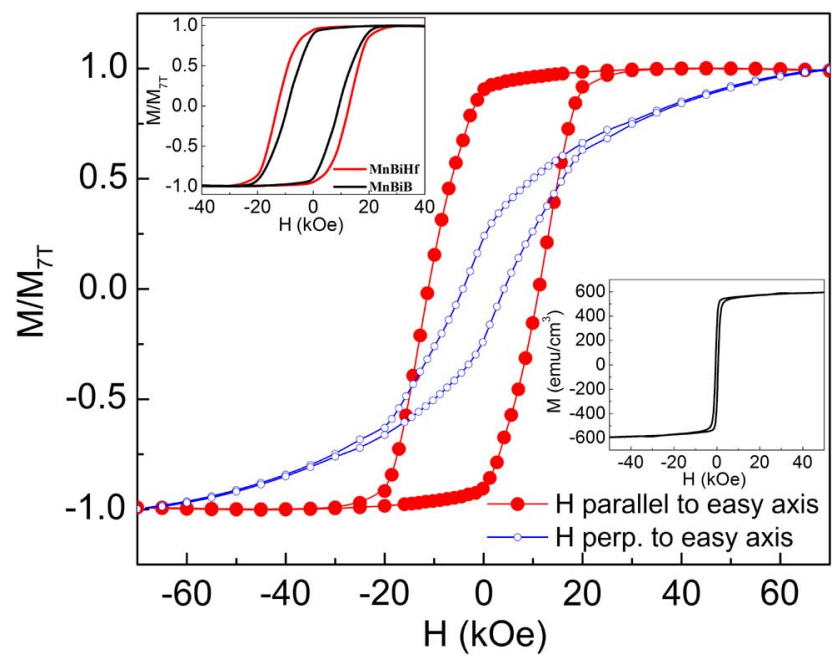

Fig. 4. Room-temperature $\mathrm{M}(\mathrm{H})$ hysteresis curves for magnetic-field aligned $\mathrm{Mn}_{54} \mathrm{Bi}_{46}$ powder. The top-left inset shows the easy-axis $\mathrm{M}(\mathrm{H})$ curves for the $\mathrm{Hf}$ and $\mathrm{B}$ doped samples aligned in a magnetic field and the bottom-right inset shows the $\mathrm{M}(\mathrm{H})$ curve of the mechanically milled powder.

As mentioned above, $\mathrm{MnBi}$ shows a high perpendicular anisotropy and also a large coercivity in thin films. This is because the easy axis of magnetization is along the c-axis, which is perpendicular to the film plane in the textured films. In order to align the particles in a magnetic field, we mechanically crushed the ribbons into fine powder in acetone and mixed with epoxy resin. A thin layer of this dilute mixture was placed on a clean silicon piece and allowed to set in a perpendicular magnetic field of about $9 \mathrm{kOe}$. Acetone is expected to reduce the oxidation of the samples during milling but x-ray diffraction patterns of the powder samples show that the oxidation was not completely prevented by acetone [13]. Fig. 4 shows the easy-axis and hard-axis $\mathrm{M}(\mathrm{H})$ loops of the magnetic-field aligned $\mathrm{Mn}_{54} \mathrm{Bi}_{46}$ sample measured at room temperature where the easy-axis $\mathrm{M}(\mathrm{H})$ loop is wide and almost rectangular. The easy-axis $\mathrm{M}(\mathrm{H})$ loops of all other $\mathrm{MnBi}$ :X samples are also almost rectangular and there is a clear anisotropy between the easy-axis and hard-axis $\mathrm{M}(\mathrm{H})$ loops. Easy-axis $\mathrm{M}(\mathrm{H})$ loops of Hf- and B-doped samples are shown in the top-left inset of Fig. 4. However, the fairly wide hard-axis $\mathrm{M}(\mathrm{H})$ loop suggests that a substantial fraction of the particles are still not aligned with their c-axis parallel to the applied magnetic field. Magnetic field alignment has produced a large gain in both the coercivity and squareness ratio $\left(\mathrm{M}_{\mathrm{r}} / \mathrm{M}_{\mathrm{s}}\right)$ of $\mathrm{MnBi}$ and $\mathrm{MnBi}$ :X samples. As shown in Fig. 3, the easy-axis coercivities of most of the samples are in the range of $10 \mathrm{kOe}$ with a maximum $\mathrm{Hc}$ of $13 \mathrm{kOe}$ in Hf doped sample. Furthermore, the ratio $M_{r} / M_{s}$ is greater than $90 \%$ for all the aligned $\mathrm{MnBi}: \mathrm{X}$ samples. We note that the values of $M_{7 \mathrm{~T}}$ measured in the powder samples are slightly smaller than the values measured in the corresponding ribbons. For example, the $\mathrm{M}_{7 \mathrm{~T}}$ measured in $\mathrm{Mn}_{54} \mathrm{Bi}_{46}$ powder is about $10 \%$ lower than the value measured in the ribbon [see the bottom-right inset of Fig. 4].

For $\mathrm{MnBi}$, the critical single-domain diameter in the demagnetized state $\left(\mathrm{d}_{\mathrm{c}}\right)$ calculated using the Kittel formula $\mathrm{d}_{\mathrm{c}}=$ $(18 \gamma \times 4 \pi) / \mathrm{J}_{\mathrm{s}}^{2}$, where $\gamma=4\left(\mathrm{AK}_{1}\right)^{1 / 2}$ is the domain wall energy, is about $0.42 \mu \mathrm{m}$. We have used the magnetic exchange energy $\mathrm{A}=1.2 \times 10^{-6} \mathrm{ergs} / \mathrm{cm}$ [4], the uniaxial anisotropy energy $\mathrm{K}_{1}=9 \mathrm{Mergs} / \mathrm{cm}^{3}$ [2] and the saturation polarization $\mathrm{J}_{\mathrm{s}}=4 \pi \mathrm{Ms}=8.4 \mathrm{kG}$ [8] for this calculation. This suggests 


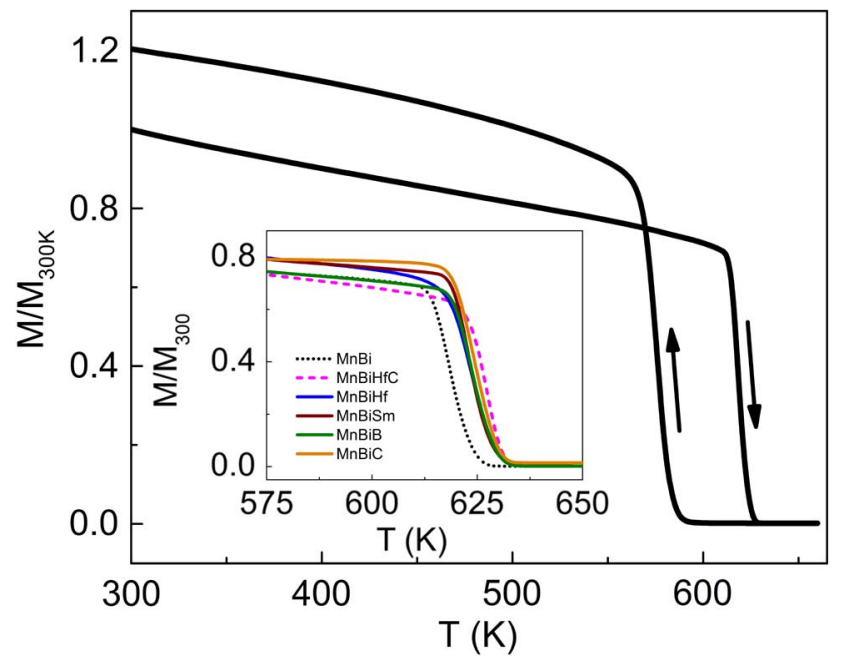

Fig. 5. Temperature dependence of magnetization $\mathrm{M}(\mathrm{T})$ of $\mathrm{Mn}_{54} \mathrm{Bi}_{46}$ ribbon measured at $\mathrm{H}=1 \mathrm{kOe}$ during heating from room temperature to $750 \mathrm{~K}$ and cooling from $750 \mathrm{~K}$ to room temperature. The inset shows the $\mathrm{M}(\mathrm{T})$ curves of $\mathrm{MnBi}$ X ribbons measured at $1 \mathrm{kOe}$ during heating.

that the average grain sizes in all of these $\mathrm{MnBi}$ X ribbons are within the critical single-domain diameter range. However, all the aligned particles are not expected to be single domain, because the mechanical milling is not sufficient to crush the ribbons into particles as small as few tenths of a micrometer in size. This is surprising that the milled powder was well aligned in the magnetic field, although the particles contain thousands of grains. This suggests that the particles are locally textured and the applied magnetic field easily rotates the particles with their easy axis along the field direction.

As shown in Fig. 5, $\mathrm{Mn}_{54} \mathrm{Bi}_{46}$ ribbons undergo a coupled structural and magnetic phase transition from a ferromagnetic low-temperature phase (LTP) to a paramagnetic high-temperature phase (HTP) at $620 \mathrm{~K}$ when the sample is heated from room temperature. When the sample is cooled from $750 \mathrm{~K}$, the sample transforms from HTP to LTP at $580 \mathrm{~K}$. The phase transition temperatures were determined from the extrapolation of the straight portion of the $M$ versus $1 / T$ curves. These values are very close to the transition temperatures $(633 \mathrm{~K}$ for LTP to HTP and $603 \mathrm{~K}$ for HTP to LTP) determined from the neutron diffraction studies of bulk MnBi [14]. The phase-transition temperature has increased by about $5 \mathrm{~K}$ due to impurity doping [see the inset of Fig. 5]. This indicates that the impurity doping has produced some modifications in the MnBi crystal lattice, which is consistent with our $\mathrm{X}$-ray diffraction studies.

\section{CONCLUSIONS}

In summary, we prepared $\mathrm{MnBi}$ ribbons doped with various impurity elements including B, C, Fe, Hf, Sm and Tb using arcmelting, melt-spinning and annealing. Magnetic properties of MnBi ribbons are very sensitive to the external impurity doping. Most of the samples show increase in coercivity and decrease in magnetization and anisotropy energy due to impurity doping. However, the sample doped with B shows significant increase in anisotropy energy and the sample co-doped with Hf and C shows a moderate increase in magnetization. The measured saturation magnetization is as high as $686 \mathrm{emu} / \mathrm{cm}^{3}$ in $\mathrm{Hf}$ and $\mathrm{C}$ co-doped sample with a maximum coercivity of $13 \mathrm{kOe}$ in the Hf-doped sample. All the MnBi:X samples show coupled structural and magnetic phase transitions around $625 \mathrm{~K}$ which is about $5 \mathrm{~K}$ higher than that of undoped $\mathrm{MnBi}$. The observed magnetic properties are explained as the consequences of the disorder and competing ferromagnetic and antiferromagnetic interactions.

\section{ACKNOWLEDGMENT}

This research is supported by the DOE under Grant DE-F602-04ER46152 (DJS, RS), the NSF MRSEC under Grant NSF-DMR-0820521 (PK), the DOE ARPA-E under Grant DE-AR0000046 (JES) and NCMN (VRS). We would like to thank Y. Huh for useful discussions.

\section{REFERENCES}

[1] N. Jones, "The pull of stronger magnets," Nature, vol. 472, pp. 22-23, 2011.

[2] J. M. D. Coey, "Hard magnetic materials: A perspective," IEEE Trans. Magn., vol. 47, pp. 4671-4681, 2011.

[3] T. Chen and W.E. Stutis, "The phase transformation and physical properties of the $\mathrm{MnBi}$ and $\mathrm{Mn}_{1.08} \mathrm{Bi}$ compounds," IEEE Trans. Magn., vol. 10, pp. 581-586, 1974.

[4] U. Rüdinger, G. Güntherodt, P. Fumagalli, V. Thomas, S. S. P. Parkin, and A. D. Kent, "Micromagnetism and high temperature coercivity of MnBi/Al multilayers," J. Appl. Phys., vol. 88, pp. 4221-4225, 2000.

[5] P. Kharel, P. Thapa, P. Lukashev, R. F. Sabirianov, E. Y. Tsymbal, D. J. Sellmyer, and B. Nadgorny, "Transport spin polarization of high Curie temperature MnBi films," Phys. Rev. B, vol. 83, pp. 024415-024416, 2011.

[6] P. Kharel, R. Skomski, P. Lukashev, R. Sabirianov, and D. J. Sellmyer, "Spin correlations and Kondo effect in a strong ferromagnet," Phys. Rev. B, vol. 84, pp. 014431-01443, 2011.

[7] P. Kharel, R. Skomski, and D. J. Sellmyer, "Spin correlations and electron transport in MnBi:Au films," J. Appl. Phys., vol. 109, pp. 07B709-07B709-3, 2011.

[8] J. B. Yang, Y. B. Yang, X. G. Chen, X. B. Ma, J. Z. Han, Y. C. Yang, S. Guo, A. R. Yan, Q. Z. Huang, M. M. Wu, and D. F. Chen, "Anisotropic nanocrystalline $\mathrm{MnBi}$ with high coercivity at high temperature," Appl. Phys. Lett., vol. 99, pp. 082505-082505-3, 2011.

[9] R. Skomski and J. M. D. Coey, Permanent Magnetism. Bristol: Institute of Physics, 1999, p. 136.

[10] G. Hadjipanayis and D. J. Sellmyer, "Rare-earth-rich metallic glasses. I. Magnetic hysteresis," Phys. Rev. B, vol. 23, pp. 3349-3354, 1981.

[11] C. Neise, S. Schönecker, M. Richter, K. Koepernik, and H. Eschrig, "The effect of chemical disorder on the magnetic anisotropy of strained Fe-Co films," Phys. Status Solidi B, vol. 248, pp. 2398-2403, 2011.

[12] P. Kharel, X. Z. Li, V. R. Shah, N. Al-Aqtash, K. Tarawneh, R. F. Sabirianov, R. Skomski, and D. J. Sellmyer, "Structural, magnetic, and electron transport properties of MnBi:Fe thin films," J. Appl. Phys., vol. 111, pp. 07E326-07E326-3, 2012.

[13] P. Kharel, V. R. Shah, X. Z. Li, W. Y. Zhang, R. Skomski, J. E. Shield, and D. J. Sellmyer, "Structural and Magnetic Properties of Pr-alloyed MnBi Nanostructures," J. Phys. D: Appl. Phys., vol. 46, p. $095003-$ 095003-7, 2013

[14] B. W. Roberts, "Neutron diffraction study of the structures and magnetic properties of manganese bismuthide," Phys. Rev. B, vol. 104, pp. 607-616, 1956. 